\title{
Endoscopic ultrasound-guided pancreaticojeju- nostomy with a forward-viewing echoendoscope as a treatment for stenotic pancreaticojejunal anastomosis
}

A 59-year-old man presented with repeated episodes of pancreatitis. His medical history included a Whipple resection for gastric carcinoma at the age of 54. A computed tomography scan showed a sac-like enlargement of the main pancreatic duct, which had obviously developed over a period of time ( $\bullet$ Fig. 1 ).

We first attempted to perform endoscopic retrograde pancreatography (ERCP). This identified the site of the anastomosis; however, we were unable to cannulate the pancreatic duct. Therefore, we decided to perform endoscopic ultrasound (EUS)-guided pancreaticojejunostomy. The procedure was carried out with a forward-viewing echoendoscope (TGFUC260J; Olympus, Tokyo, Japan). After reaching the anastomosis site, we observed the dilated pancreatic duct by means of EUS. The pancreatic duct was then punctured with a 19-gauge needle (EchoTip Ultra; Cook Medical, Bloomington, Indiana, USA) ( Fig. 2 ). After the puncture procedure, contrast medium was injected to confirm that the needle was located in the pancreatic duct. Next, a guidewire (VisiGlide 2; Olympus) was inserted into the pancreatic duct through the puncture needle, and the needle was then removed whilst the guidewire remained in place ( $\bullet$ Fig.3). The fistula was subsequently dilated in a stepwise manner with a 4-Fr tapered tip cannula (StarTip V; Olympus) and 6-, 7-, and 9-Fr dilation catheters (Soehendra biliary dilation catheters; Cook Medical), which allowed us to place a plastic stent (QuickPlace V, 7.2-Fr; Olympus) across the pancreaticojejunostomy ( $\bullet$ Fig.4).

Recently, EUS-guided transgastric puncture has been reported to be a useful treatment for pancreatic duct obstruction [1 - 3], although the technique carries the risk of pancreatic juice leakage. On the

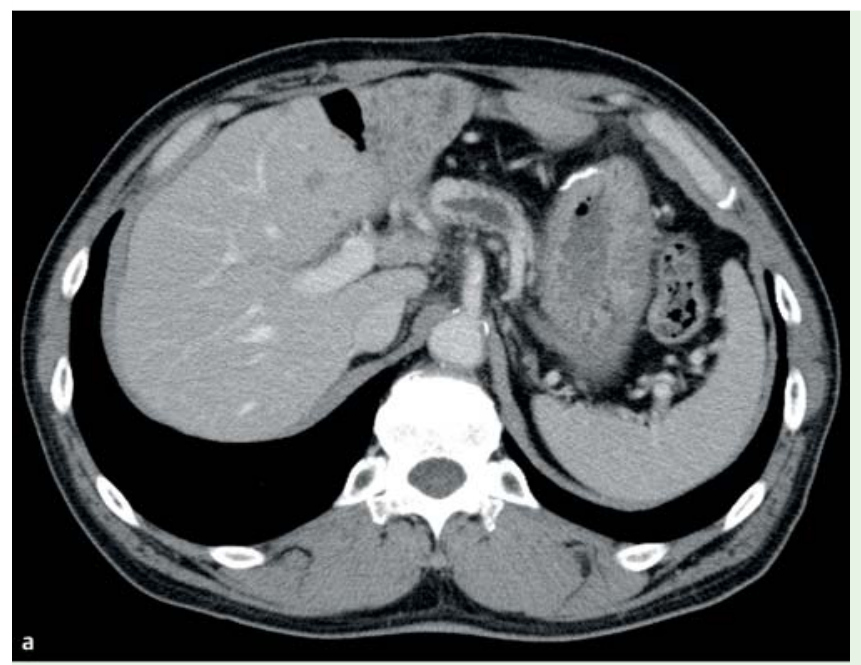

Fig. 1 A 59-year-old man, who had undergone Whipple resection for gastric carcinoma 5 years previously, presented with repeated episodes of pancreatitis. a A computed tomography (CT) 2 years previously showed mild dilation of the main pancreatic duct. b The dilation of the main pancreatic duct had obviously developed over a period of time.
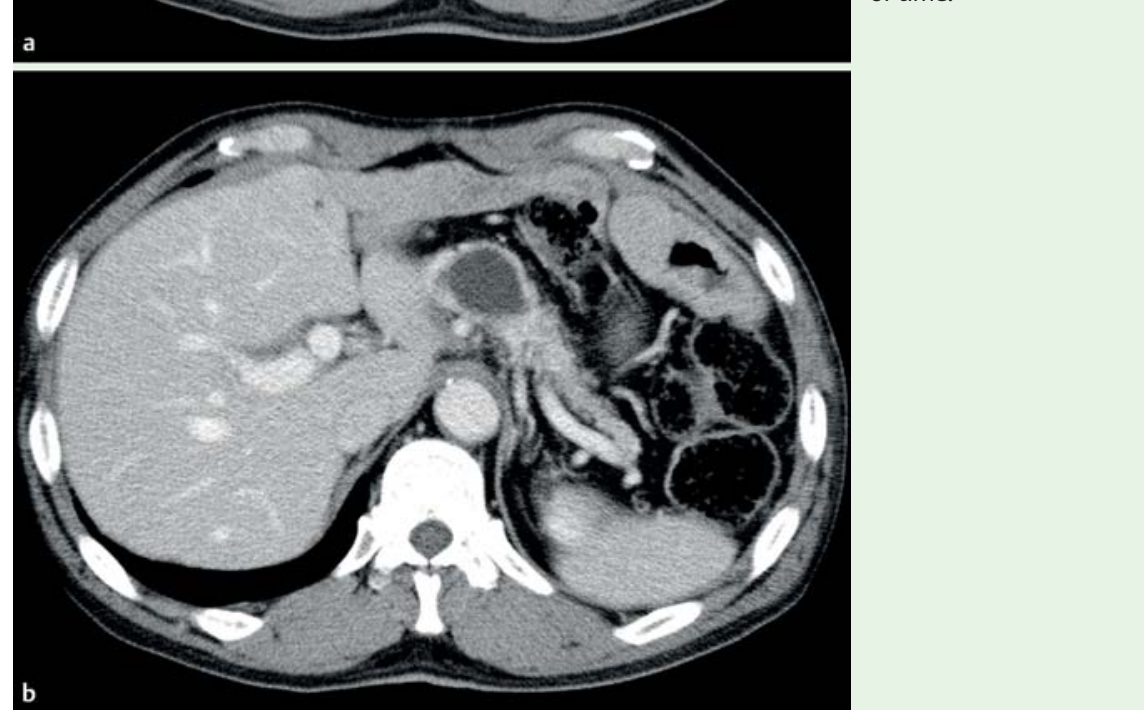

other hand, EUS-guided retrograde panEndoscopy_UCTN_Code_TTT_1AS_2AD creatic stent placement using a conventional echoendoscope has also been reported [4]; however this was associated with difficulties in creating and dilating the fistula. Therefore, we consider the use of a forward-viewing echoendoscope to be a more feasible approach to performing EUS-guided pancreaticojejunostomy.

\section{Competing interests: None}




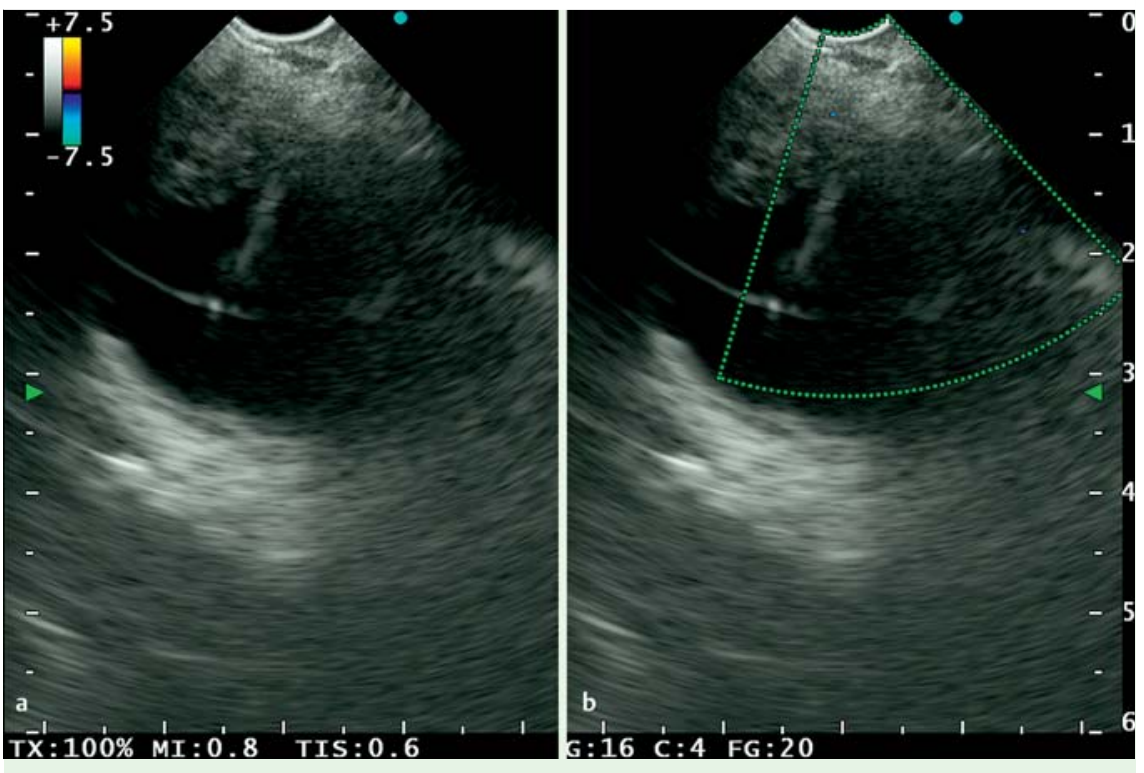

Fig. 2 The dilated main pancreatic duct was punctured with a 19-gauge needle.

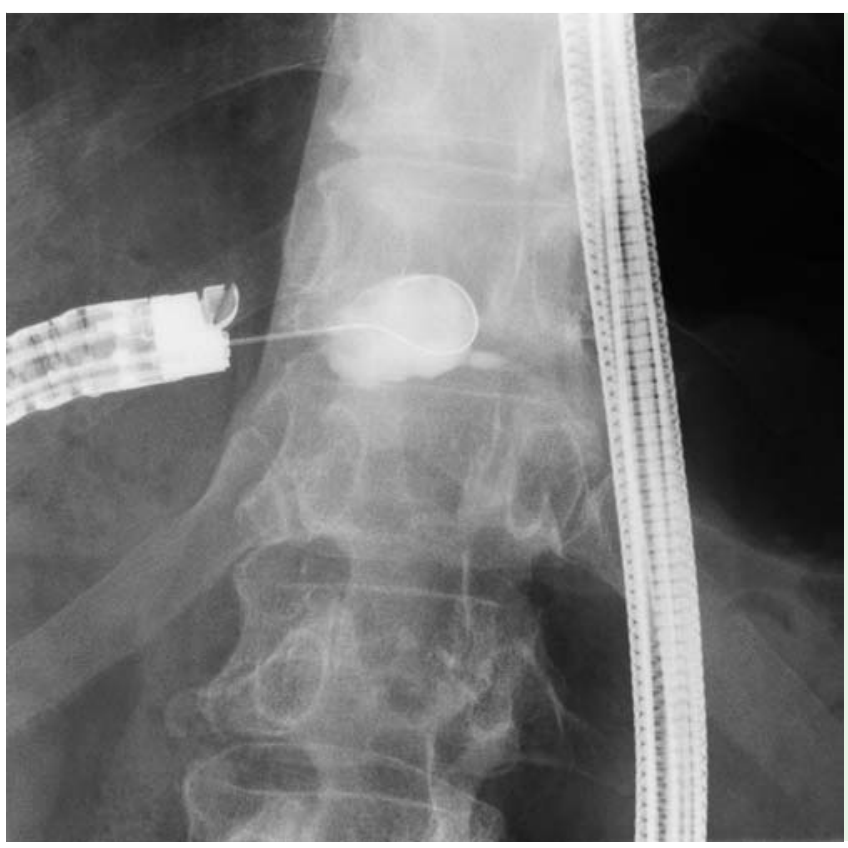

Fig. 3 Contrast medium was injected, and a guidewire was inserted into the pancreatic duct.
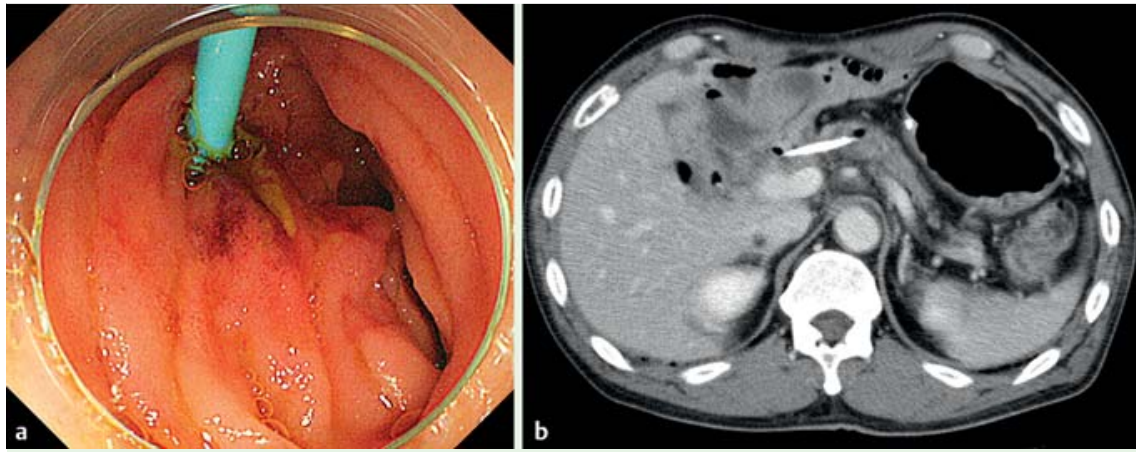

Fig. 4 A plastic stent was placed across the pancreaticojejunostomy: $\mathbf{a}$ endoscopic view; $\mathbf{b}$ computed tomography $(\mathrm{CT})$ image.

\section{So Nakaji, Nobuto Hirata, Toshiyasu Shiratori, Masayoshi Kobayashi, Kenji Yamauchi}

Department of Gastroenterology, Kameda Medical Center, Kamogawa, Chiba, Japan

\section{References}

1 Bataille L, Deprez P. A new application for therapeutic EUS: main pancreatic duct drainage with a "pancreatic rendezvous technique". Gastrointest Endosc 2002; 55 : $740-743$

2 Kinney TP, Li R, Gupta $K$ et al. Therapeutic pancreatic endoscopy after Whipple resection requires rendezvous access. Endoscopy 2009; 41: 898-901

3 Itoi T, Kikuyama M, Ishii K et al. EUS-guided rendezvous with single-balloon enteroscopy for treatment of stenotic pancreaticojejunal anastomosis in post-Whipple patients (with video). Gastrointest Endosc 2011; 73: 398-401

4 Matsubayashi H, Kishida Y, Shinjo $K$ et al. Endoscopic ultrasound-guided retrograde pancreatic stent placement for the treatment of stenotic jejunopancreatic anastomosis after a Whipple procedure. Endoscopy 2013; 45: E435-436

\section{Bibliography}

DOI http://dx.doi.org/

10.1055/s-0034-1391245

Endoscopy 2015; 47: E41-E42

(c) Georg Thieme Verlag KG

Stuttgart · New York

ISSN 0013-726X

\section{Corresponding author}

\section{So Nakaji, MD}

929, Higashi-cho

Kamogawa, Chiba 296-8602

Japan

Fax: +81-4-70991191

nakajisou1978@yahoo.co.jp 